\title{
Measurement of Optical Pulsewidth in the Picosecond Regime Using a Non-linear Fiber and Power Meter
}

\author{
Umair Ahmed Korai ${ }^{1,2}$, Zifei Wang ${ }^{3}$, Cosimo Lacava ${ }^{4}$, Lawrence R. Chen ${ }^{3}$, Michael J. Strain ${ }^{5}$, Ivan Glesk ${ }^{1}$ \\ ${ }^{1}$ Dept. of Electronic and Electrical Engineering, University of Strathclyde, Glasgow G1 1XW, UK \\ ${ }^{2}$ Dept. of Telecommunication Engineering, Mehran University of Engineering and Technology, Jamshoro, 76062, Pakistan \\ ${ }^{3}$ Dept. of Electrical and Computer Engineering, McGill University, Montreal, H3A OE9, Canada \\ ${ }^{4}$ Optoelectronics Research Centre, University of Southampton, Southampton SO17 1BJ, UK \\ ${ }^{5}$ Institute of Photonics, Dept. of Physics, University of Strathclyde, Glasgow G1 1RD, UK \\ Author e-mail address: Umair.korai@faculty.muet.edu.pk
}

\begin{abstract}
:
A technique for the characterization of picosecond pulse widths is presented, based a non-linear optical fiber loop mirror and power meter measurement. Pulse-widths in the 2-10ps range are successfully recovered with a resolution of $0.25 \mathrm{ps}$.

OCIS codes: (190.0190) Nonlinear optics; (190.5530) Pulse propagation and temporal solitons; (140.3538) Lasers, pulsed
\end{abstract}

\section{Introduction}

Short laser pulses are widely deployed in applications in optical communications, medicine, and industry [1]. A number of well-established techniques for full characterization of both the pulse amplitude and phase of laser pulses have been developed. Among the most known include frequency resolved optical gating (FROG) [2] and spectral phase interferometry for direct electric-field reconstruction of ultrashort optical pulses (SPIDER) [3]; however, these techniques require either complex experimental setups or expensive equipment. In some cases, however, retrieval of the pulse width is sufficient, for example, in all-optical performance monitoring in optical communications applications and dispersion monitoring [4]. High-speed photodetectors and sampling oscilloscopes as well as intensity auto-correlators or cross-correlators can be used in a laboratory environment for this purpose. As a compact, all-fiber alternative we have developed a simple method which employs a fiber amplifier, nonlinear optical loop mirror (NOLM), based on highly nonlinear fiber (HNLF), and an optical power meter. The experimental demonstration successfully retrieved the temporal width of an optical signal in a range from 2 to $10 \mathrm{ps}$ with resolution of $0.25 \mathrm{ps}$ assuming the signal has sech ${ }^{2}$ envelope and slowly varying phase internal structure. The experimental result shows an excellent agreement with the simulations. Simulations show that by varying the fiber length or amplifier saturated power level, the measureable pulse range can be expanded to 0.25 to $90 \mathrm{ps}$.

\section{Experimental verification of simulation results}

The experimental setup was built to verify the proposed concept, device performance and its operation range. Fig. 1(a) shows the NOLM in a cross-phase modulation (XPM) configuration. Coarse wavelength add/drop multiplexers $\left(\mathrm{CWDM}_{1}\right.$ and $\left.\mathrm{CWDM}_{2}\right)$ were used to couple the pulsed measurement signal from a mode-locked fiber laser (MLL) in and out of the NOLM. An EDFA with a saturated power of $17 \mathrm{dBm}$ was used to sweep the average power of the signal.

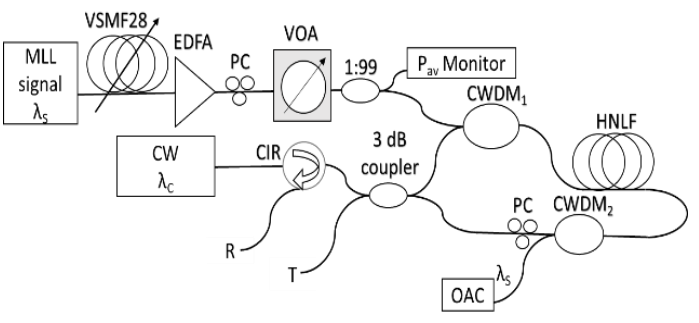

(a)

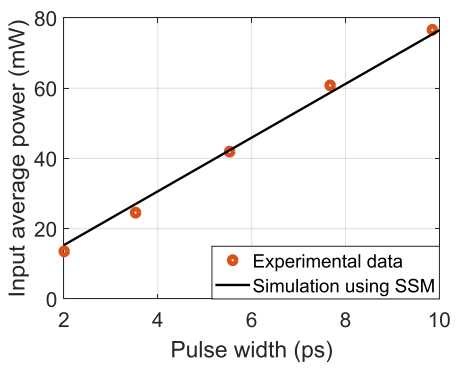

(b)

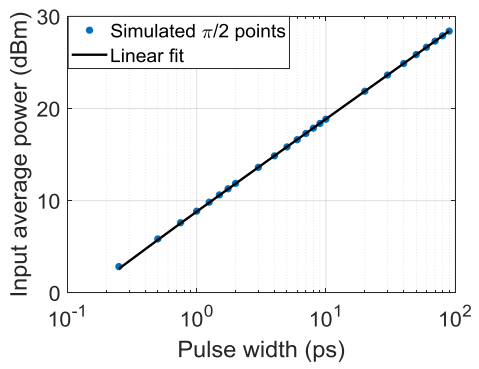

(c)

Fig. 1(a): Experimental setup for measuring short optical pulse widths; b) Device calibration based on simulation results and experimental data; c) Simulated device operation range considering the length of HNLF as $100 \mathrm{~m}$. Where, $M L L$ - mode locked fiber laser, $H N L F-$ highly non-linear optical fibre, $C I R$ - optical circulator, $E D F A$ - erbium doped fiber amplifier, $C W D M$ - coarse wavelength division multiplexer, $P C$ - polarization controller, VSMF28 - variable length of single mode fiber (SMF-28); OAC - optical autocorrelator. 
The MLL, a PriTel fiber laser at $10 \mathrm{GHz}$, was used to produce $\tau_{\mathrm{MLL}}=2$ ps pulses (spectral width of $1.4 \mathrm{~nm}$, central wavelength $\lambda_{\mathrm{S}}=1550.7 \mathrm{~nm}$ ). For the purpose of calibration and testing, a varying pulse width of $3.52 \mathrm{ps}, 5.5 \mathrm{ps}, 7.7$ ps and 9.8 ps was achieved by adding additional lengths of $100 \mathrm{~m}, 200 \mathrm{~m}, 300 \mathrm{~m}$ and $450 \mathrm{~m}$ fiber cables, respectively into the setup, after the MLL. After passing a variable optical attenuator (VOA) a $1 \%$ power tap was introduced for MLL average power $\left(P_{\mathrm{av}}\right)$ monitoring. A low power continuous wave $(\mathrm{CW})$ probe was generated using an Agilent $81989 \mathrm{~A}$ tunable laser at $\lambda_{\mathrm{CW}}=1530 \mathrm{~nm}$ with a power of $5 \mathrm{~mW}$. The NOLM transmitted $\left(P_{\mathrm{T}}\right)$ and reflected $\left(P_{\mathrm{R}}\right)$ average powers were measured at transmission $(T)$ and reflection $(R)$ ports, respectively. For calibration purposes, the optical pulse widths were also measured using a FR-103XL optical auto-correlator. We found by simulations that the optical pulsewidth generated by MLL can be recovered from measurements of the average powers $P_{\mathrm{av}}, P_{\mathrm{R}}$, and $P_{\mathrm{T}}$. In our simulations we assumed the envelope function of the optical pulses is $\operatorname{sech}^{2}$. The nonlinear phase shift was calculated by solving the nonlinear Schrodinger equation for cross phase modulation (XPM) using the split step method [5]. These simulations were based on the fiber specifications used in the experiment, i.e. length $\left(L_{\mathrm{HNLF}}=100 \mathrm{~m}\right)$, gamma $\left(\gamma=10.8 \mathrm{~W}^{-1} \cdot \mathrm{km}^{-1}\right)$, dispersion $(D=0.11 \mathrm{ps} / \mathrm{nm} / \mathrm{km})$, dispersion slope $\left(S=0.016 \mathrm{ps} / \mathrm{nm}^{2} / \mathrm{km}\right)$, absorption coefficient $(\alpha=0.9 \mathrm{~dB} / \mathrm{km})$ and splice loss (HNLF to SMF28) as $0.2 \mathrm{~dB}$. The $P_{\mathrm{av}}$ can induce a phase shift which creates switching between the $R$ and $T$ ports. The figure of merit for the system is defined as the average power in the signal required to induce a $\pi / 2$ phase switching between the $R$ and $T$ ports. The simulated and measured $\pi / 2$ switching power points with respect to pulsewidth of an optical signal are shown in Fig.1(b). The input pulse widths were successfully characterized, with a resolution of $0.25 \mathrm{ps}$, based on the average power meter accuracy.

\section{Device operation range}

In order to measure the temporal width of an optical pulse, the setup described above is required to define the $\pi / 2$ switching power point. This therefore puts a limitation on the measurement range of the system that depends on the available saturation power of the EDFA and the length of HNLF loop. Assuming the dispersion length is longer than the nonlinear length, the nonlinear phase shift for XPM is given as $\emptyset_{\boldsymbol{X P M}}=\frac{\mathbf{2} \gamma \boldsymbol{P}_{\boldsymbol{a v}} \boldsymbol{L}_{\boldsymbol{e f f}}}{\boldsymbol{f \tau} \boldsymbol{\tau}}$, where $\gamma$ is the nonlinear coefficient, $f$ is the laser repetition rate, $\tau$ is the unknown pulse width of the laser signal, and $L_{\text {eff }}$ is the effective length of the HNLF and given as $\boldsymbol{L}_{\boldsymbol{e f f}}=\frac{1-\exp \left(-\alpha \boldsymbol{L}_{\boldsymbol{H} N L F}\right)}{\alpha}$ [5]. When considering $\emptyset_{X P M}$, it is clear that the operation range for measuring an optical pulsewidth can be set by adjusting the HNLF length $\left(L_{\mathrm{HNLF}}\right)$ and the EDFA saturated power, i.e. $P_{\text {av }}$. By using XPM simulations, the dynamic range of the measurement system can by estimated for practically achievable lower and upper limits for the following parameters. We considered the $f=10 \mathrm{GHz}$ and $L_{\mathrm{HNLF}}=100 \mathrm{~m}$ and increase the EDFA saturated output power. From Fig. 1(c), it can be observed that by using 30 dBm EDFA, pulse widths up to 90 ps can be measured. The lower measurement bound is set by the resolution of the method at 0.25 ps. A tradeoff can be made between the available EDFA saturated power and the length of HNLF for calculating a specific optical pulse width. The device can be also reconfigured for pulse width measurements beyond a 100 ps range, however for this regime, high speed electronics may be a more attractive option.

\section{Conclusion}

We presented an all-fiber non-linear interferometric method for determining the optical pulsewidth from measuring the average optical power using an optical power meter. The approach is applicable where sech ${ }^{2}$ pulse envelopes can be assumed and the internal phase structure is slowly varying. Pulse widths in the range of 2 to 10 ps have been measured experimentally with a resolution of $0.25 \mathrm{ps}$ and show excellent agreement with simulations. The range can further be extended from 0.25 to 90 ps by selecting different physical parameters of the setup. The method may find use in distributed networks or as a bolt-on to optical systems using ultra-short pulse sources. This fiber device can be also implemented as an integrated NOLM or Mach-Zehnder interferometer using silicon on insulator platform where the nonlinear coefficient is very high, i.e. $307(\mathrm{Wm})^{-1}[6]$, hence help with device miniaturization and power.

This work is supported by the Commonwealth Scholarship Commission United Kingdom and the European Union Horizon 2020 research and innovation program under the Marie Skłodowska-Curie Grant Agreement No. 734331

\section{References}

[1] M. E. Fermann, I. Hartl, "Ultrafast fibre lasers," Nat. Photonics 7, 868-874 (2013).

[2] R. Trebino, D. J. Kane, "Using phase retrieval to measure the intensity and phase of ultrashort pulses: frequency-resolved optical gating," J. Opt. Soc. Am. A 10(5), $1101-1111$ (1993).

[3] A. Pasquazi, et al., "Sub-picosecond phase-sensitive optical pulse characterization on a chip," Nat. photonics 5, 618 - 623 (2011).

[4] J.Y. Yang, et al., "All-Optical Chromatic Dispersion Monitoring for Phase-Modulated Signals Utilizing Cross-Phase Modulation in a Highly

Nonlinear Fiber," IEEE Photonics Technology Lett. 20 (19), 1642 - 1644 (2008)

[5] G. P. Agrawal, "Nonlinear Fiber Optics," 4th ed. Academic Press, Boston (2007).

[6] J. Leuthold, C. Koos, W. Freude, "Nonlinear silicon photonics," Nat. Photonics 4, 535-544 (2010) 